\title{
Photoluminescence quenching mechanisms in type II InAs/GaInSb QWs on InAs substrates
}

\author{
M. Dyksik ${ }^{1}$ M. Motyka ${ }^{1} \cdot$ M. Kurka ${ }^{1} \cdot$ K. Ryczko ${ }^{1} \cdot$ \\ M. Dallner ${ }^{2}$ S. Höfling ${ }^{2,3} \cdot$ M. Kamp ${ }^{2}$ G. Sęk ${ }^{1}$ • \\ J. Misiewicz ${ }^{1}$
}

Received: 6 May 2016/Accepted: 21 July 2016/Published online: 28 July 2016

(C) The Author(s) 2016. This article is published with open access at Springerlink.com

\begin{abstract}
Optical properties of AlSb/InAs/GaInSb/InAs/AlSb quantum wells (QWs) grown on an InAs substrate were investigated from the point of view of room temperature emission in the mid- and long-wavelength infrared ranges. By means of two independent techniques of optical spectroscopy, photoreflectance and temperature-dependent photoluminescence, it was proven that the main process limiting the performance of such InAs substrate-based type II structures is related to the escape of carriers from the hole ground state of the QW. Two nonradiative recombination channels were identified. The main process was attributed to holes tunneling to the valence band of the GaAsSb spacing layer and the second one with trapping of holes by native defects located in the same layer.
\end{abstract}

Keywords Fourier-transform spectroscopy $\cdot$ Mid-infrared photoluminescence $\cdot$ Type II quantum wells $\cdot$ Localized states

This article is part of the Topical Collection on TERA-MIR: Materials, Generation, Detection and Applications.

Guest Edited by Mauro F. Pereira, Anna Wojcik-Jedlinska, Renata Butkute, Trevor Benson, Marian Marciniak and Filip Todorov.

M. Dyksik

mateusz.dyksik@pwr.edu.pl

1 Laboratory for Optical Spectroscopy of Nanostructures, Division of Experimental Physics, Faculty of Fundamental Problems of Technology, Wrocław University of Science and Technology, Wybrzeże Wyspiańskiego 27, 50-370 Wrocław, Poland

2 Technische Physik, Wilhelm-Conrad-Röntgen-Research Center for Complex Material Systems, University of Würzburg, Am Hubland, 97074 Würzburg, Germany

3 SUPA, School of Physics and Astronomy, University of St. Andrews, North Haugh, St. Andrews KY16 9SS, UK 
Interband cascade lasers (ICLs) (Meyer et al. 1995; Yang 1995), emitting at room temperature in the continuous-wave operation mode, are of interest for applications in optical gas sensors (Bauer et al. 2010), utilized e.g. for human breath analysis (Tittel 2010) or detection of harmful or toxic gases, like formaldehyde (Lundqvist et al. 2012), methane (Dong et al. 2016) or nitric oxide (Von Edlinger et al. 2014). ICLs have been shown to be efficient laser sources, with high power operation at room temperature (Jiang et al. 2014; Kim et al. 2012) and low threshold currents, resulting in low power consumption (Vurgaftman et al. 2011). Nevertheless, there is still a huge demand for new generation devices emitting in the long-wavelength infrared (LWIR) range, in particular to detect higher order hydrocarbons. Such ICL devices are typically based on InAs substrates instead of GaSb, resulting in employment of plasmon-enhanced claddings (Ohtani and Ohno 2002; Tian et al. 2012). It has been shown that lasers based on such concept operate at temperatures above ambient, up to $377 \mathrm{~K}$, while lasing at $\sim 5 \mu \mathrm{m}$ in pulsed mode. The LWIR emission, including the nowadays record of $11 \mu \mathrm{m}$ ( $\mathrm{Li}$ et al. 2015), is mostly delivered by multimode devices operating at low temperatures in pulsed-mode, limiting their possible application in optical gas sensors. The longest emitted wavelength among the devices operating at room temperature has been shown to be $7 \mu \mathrm{m}$ (Dallner et al. 2015) in pulsed mode, thus there is still plenty of room for further improvements and development of ICLs grown on InAs substrates, especially for those operating at ambient temperature in a continuous-wave mode. Therefore knowledge on carrier loss mechanisms reducing the maximum operation temperature is of high interest.

In this paper, we have studied type-II W-design AlSb/InAs/GaInSb/InAs/AlSb quantum wells (QWs) grown on an InAs substrate, designed to emit at ambient temperature in a broad spectral range from the mid- $(\sim 5 \mu \mathrm{m})$ to long-wavelength (beyond $8 \mu \mathrm{m}$ ) infrared. We have measured the temperature dependent photoluminescence in order to determine the main carrier escape channels, which are usually being the main factors limiting the device performance. The obtained results have been discussed and compared with previously published results regarding GaSb-based quantum wells designed for emission below $4 \mu \mathrm{m}$ (Sęk et al. 2011).

The investigated structures were grown on an (100) oriented InAs substrate, in a solid source molecular beam epitaxy system equipped with valved cracker cells for both antimony and arsenic. Four structures containing type II quantum wells as an optically active region were studied. Samples A-C were designed in a common "W-shaped" scheme, with two InAs layers for the electron confinement and one GaInSb layer for the confinement of holes. Table 1 summarizes the QWs structural parameters obtained from the analysis of high-resolution X-ray diffraction data and the growth calibration procedures. Each QW is surrounded by $2.5 \mathrm{~nm}$ thick AlSb barriers. In order to enhance the overall optical response each sample contains five such "W-like" quantum wells, separated by a $25 \mathrm{~nm}$ thick $\mathrm{GaAs}_{0.08} \mathrm{Sb}_{0.92}$ layer lattice-matched to InAs. The entire structure is terminated by the same $\mathrm{GaAs}_{0.08} \mathrm{Sb}_{0.92}$ layer.

Table 1 The layer structure of the investigated QWs

\begin{tabular}{lll}
\hline Sample name & $\begin{array}{l}\text { InAs thickness } \\
(\mathrm{nm})\end{array}$ & $\begin{array}{l}\mathrm{Ga}_{0.76} \mathrm{In}_{0.24} \mathrm{Sb} \\
\text { thickness }(\mathrm{nm})\end{array}$ \\
\hline Sample A & 2.8 & 3 \\
Sample B & 2.95 & 3 \\
Sample C & 3.1 & 3 \\
\hline
\end{tabular}


In order to measure photoluminescence (PL) and photoreflectance (PR) in a wide spectral range an evacuated Fourier-transform (FT) spectrometer Bruker Vertex 80v was used and operated in step-scan mode (Firsov and Komkov 2013; Motyka et al. 2009b; Motyka and Misiewicz 2010). A liquid-nitrogen cooled mercury cadmium telluride photodetector and a $\mathrm{KBr}$ beamsplitter were employed. For both PL and PR measurements, the pump beam was provided by a $640 \mathrm{~nm} 60 \mathrm{~mW}$ semiconductor laser diode, which was mechanically chopped at a frequency of $275 \mathrm{~Hz}$. The phase sensitive detection of the optical response was performed using a lock-in amplifier. A similar FT-based approach has been demonstrated to be an efficient tool for optical characterization in the mid-infrared spectral range of narrow-band gap materials (Hosea et al. 2005; Shao et al. 2007) as well as quantum cascade lasers (Dyksik et al. 2016; Pierscinski et al. 2014).

To calculate the electronic structure of the type-II W-design QWs we used the $8 \times 8$ k.p Hamiltonian defined for the [001] growth direction (Ryczko et al. 2013). The model includes the strain effects after (Bir et al. 1976). The carrier wavefunctions and subband energies were determined by numerically solving the Schrödinger equation and employing the finite difference method (Thomas 1995). All material parameters were taken from (Vurgaftman et al. 2001).

Figure 1a presents the band alignment of the InAs-based type II AlSb/InAs/GaInSb/ InAs/AlSb quantum well together with the latticed-matched spacing layer made of $\mathrm{GaAs}_{0.08} \mathrm{Sb}_{0.92}$. The valence band edge of such layer is approx. $100 \mathrm{meV}$ below the valence band of GaSb (see Fig. 1b). It has been shown before that GaSb-based structures with a GaSb spacing layer exhibit a nonradiative recombination channel attributed to the tunneling of holes from the QW to the valence band of the GaSb spacer (Sęk et al. 2011). This process is expected to be eliminated in the case of InAs-based structures due to a much deeper confinement potential for holes, with respect to the valence band edge of the $\mathrm{GaAs}_{0.08} \mathrm{Sb}_{0.92}$ layer.

In general, the QW emission can be quenched by (i) an escape of carriers from the QW through excited states; (ii) a direct escape of electrons (holes) from the respective QW ground state to the electron (hole) band continuum; (iii) trapping of carriers by defect states (e.g. in the barriers). In order to find out if a scenario with the first process is probable for the given structures, the absorption-like photoreflectance measurements were performed to probe the spectrum of the excited states. The second and third processes were investigated by means of the temperature resolved photoluminescence and will be discussed below yet.

Fig. 1 Band alignment for investigated type II QW with the GaAsSb a and GaSb b spacing layers

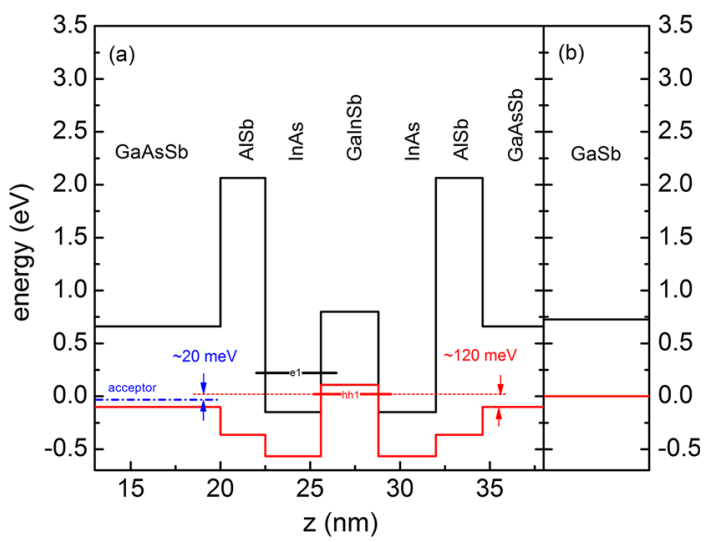


Fig. 2 Low temperature (77 K) photoreflectance spectra for AlSb/InAs/GaInSb/InAs/AlSb QWs with different InAs layers width

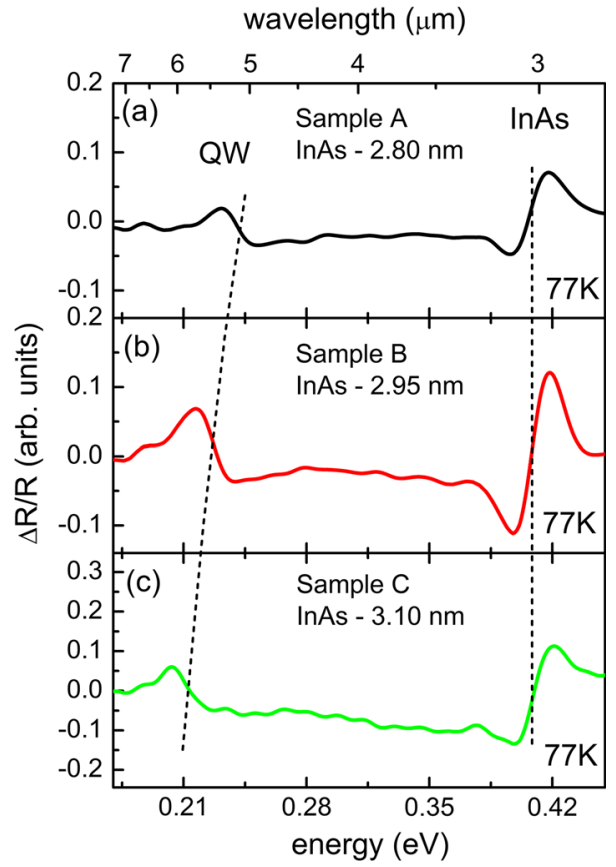

Figure 2 presents the photoreflectance spectra for the samples A, B and C, measured at a temperature of $77 \mathrm{~K}$. For each spectrum the signal at $0.41 \mathrm{eV}$ is related to the InAs band gap optical transition, whereas the signal at lower energy side is associated with the fundamental transition in the investigated quantum wells, between the first heavy hole and the first electron states. A typical energy shift due to the thickness variation of the electron confining InAs layer is visible. Similar behavior was also observed for the type II QWs grown on a GaSb substrate (Motyka et al. 2012, 2009a). In this case, widening of an InAs well from 2.8 to $3.1 \mathrm{~nm}$ allowed decreasing the transition energy from 240 to $210 \mathrm{meV}$, which translates into an almost $1 \mu \mathrm{m}$ shift in the wavelength scale. In the PR spectra no evidence of additional features related to the excited states was found between the two singularities separated by $\sim 200 \mathrm{meV}$. Although the calculations predict the presence of the second confined electron state in such a broad InAs well, its wavefunction exhibits an asymmetric nature thus the transition matrix element with the respective hole state is negligible. The process of electrons escaping from the QW through the ground and/or excited state is not relevant here, since, both states are below the QW edge by $\sim 480$ and $\sim 570 \mathrm{meV}$, respectively. At this point it is worth pointing out that according to calculations the energy distance between the first and the second electron levels is $\sim 90 \mathrm{meV}$ for samples A-C. Further widening of the InAs well in order to reach longer emission wavelengths is subsequently reducing the distance between the respective electron states, with $82 \mathrm{meV}$ for the $3.5 \mathrm{~nm}$ wide InAs well. As a result, the reasonable spacing between both states should not influence the selective injection of carriers into the upper lasing level in an operational device.

Figure 3 compares room temperature PL spectra for the three investigated samples A, B and C. The effect of the InAs layer thickness on the confinement of electrons is clearly visible, in spite of very small QW width changes. Sample A exhibits a peak of emission at 
Fig. 3 Room temperature photoluminescence for all investigated QWs, with different InAs layer thickness

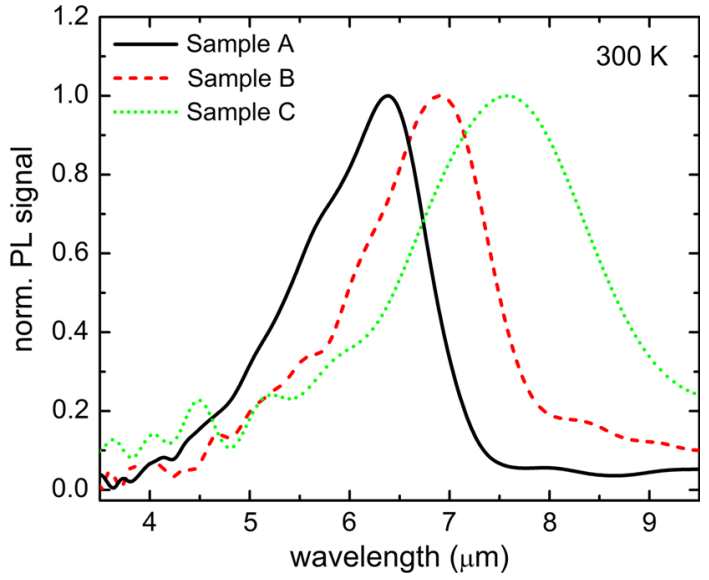

$\sim 6 \mu \mathrm{m}$, whilst sample $\mathrm{C}$ with $\sim 0.3 \mathrm{~nm}$ thicker $\mathrm{InAs}$ layer emits around $8 \mu \mathrm{m}$, i.e. reaching the long-wavelength infrared region. This might be considered as an important factor in the case of emitters in the LWIR spectral range where reduction of the fundamental transition by $10 \mathrm{meV}$ allows obtaining hundreds of nanometers shift in the emitted wavelength (Dyksik et al. 2015).

The integrated PL intensity was extracted from each individual spectrum $I(E)$ by calculating the $I(E) d E$ integral, where $E$ is the photon energy. The Arrhenius plot of the integrated PL for sample A is presented in Fig. 4. The measurements were performed in a wide range of temperatures, from 15 to $310 \mathrm{~K}$, and a total energy shift of about $40 \mathrm{meV}$ was observed, which corresponds mainly to the shrinkage of the respective band gaps with temperature. At this point it is worth to mention the sensor's temperature read-out is assumed to be identical to the sample's active region temperature since (i) the sample is mounted on the heat sink with the temperature sensor and heater just behind it and (ii) the external pump beam $\left(60 \mathrm{~mW}\right.$, spot size of $\left.9 \mathrm{~mm}^{2}\right)$ should not influence the sample temperature due to the generated power density lower than $1 \mathrm{Wcm}^{-2}$.

In order to investigate the mechanism of PL quenching, the integrated PL emission was analyzed by means of the standard Arrhenius formula:

Fig. 4 Analysis of photoluminescence signal intensities as the function of inverse temperature with activation energies presented in inset

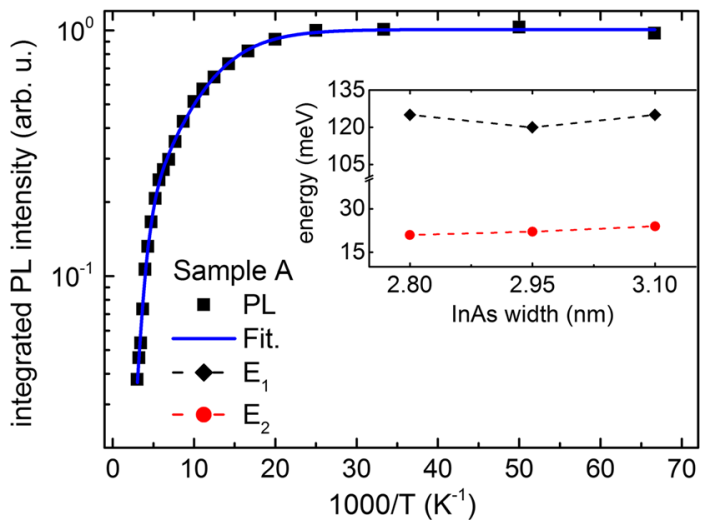




$$
I(T)=\frac{I(0)}{1+\sum_{i=1}^{n} C_{i} \exp \frac{-E_{i}}{k_{B} T}}
$$

which can be interpreted in terms of $n$ thermally activated processes, with activation energies $E_{i}$ and amplitude parameters $C_{i}$ understood as the ratio of radiative to nonradiative lifetimes (Fang et al. 2015). The solid line in Fig. 3 stands for the model from Eq. 1. In order to perform the fitting procedure, two activation energies were assumed, reflecting two independent carrier loss processes. Similar procedure was also performed for the samples $\mathrm{B}$ and $\mathrm{C}$, in order to determine the activation energies in the investigated quantum wells.

The activation energies $E_{1}$ and $E_{2}$ as a function of the InAs thickness are presented in the inset to Fig. 3. The first activation energy (black diamonds) remains nearly constant for all the investigated samples and equals to approx. $120 \mathrm{meV}$. This energy is assigned to a process of thermally activated carriers tunneling from the heavy hole state to the valence band of the $\mathrm{GaAs}_{0.08} \mathrm{Sb}_{0.92}$ spacer (see Fig. 1). Although GaAsSb and GaInSb layers are separated by a $2.5 \mathrm{~nm}$ thick AlSb barrier and $3 \mathrm{~nm}$ thick InAs layer, such tunneling process was also reported in similar type II material systems (Liu et al. 1993; Longenbach et al. 1990). In our case the calculated tunneling probability of a thermally activated hole into the valence band edge of GaAsSb equals to $1.8 \times 10^{-4}$, meaning every thousandth hole would tunnel through the barrier. The nearly constant value of $E_{l}$ is consistent with theoretical predictions since the GaInSb layer for the hole confinement has the same thickness in every measured sample, thus the energy distance between the heavy hole state and the GaAsSb valence band edge is constant.

The second process affecting the PL intensity and manifested by another activation energy $E_{2}$, which equals to $\sim 20 \mathrm{meV}$ for samples A-C, can be connected with a process of trapping holes by the low-energy native defects in GaAsSb. This unintentionally doped GaSb-based compound is always p-type due to antisite defects (Kujala et al. 2014; Suhandi et al. 2011) ( $\mathrm{Ga}$ atoms in Sb sites), providing an acceptor trapping state approx. 90-100 meV above the valence band edge, i.e. in the gap (dash-dot line in Fig. 1). In our energy scale it is about $20-30 \mathrm{meV}$ from the bottom of the confined hole subband. Although the second process is present, the ratio $\mathrm{C} 1 / \mathrm{C} 2$, reflecting the efficiency of each process, equals to $\sim 100$. Thus one can suppose that the holes are lost more effectively by the tunneling to the band edge of the neighboring spacer layer than to be trapping by the native defects in GaAsSb. Also, the density of states for the latter one is assumed to be orders of magnitude lower, which affects the probability of the tunneling-assisted trapping process of the holes.

In conclusion, we have performed optical measurements on a set of type II QW samples grown on InAs substrates emitting in the range of 5-8 $\mu \mathrm{m}$. Two channels of nonradiative recombination were identified by means of photoreflectance and temperature resolved photoluminescence measurements. The main process limiting the performance was attributed to holes escaping from the valence band well via tunneling through the AlSb barrier to the valence band of the GaAsSb spacing layer. Since the valence band of GaAsSb is located approx. $100 \mathrm{meV}$ below the respective valence band of $\mathrm{GaSb}$, the deeper potential for the confinement of holes with respect to $\mathrm{GaSb}$ is expected and manifested by the activation energy of $\sim 120 \mathrm{meV}$. This value is almost one order of magnitude higher than the value reported for GaSb-based structures, which might be beneficial in the case of operational ICL devices. The second activation energy was connected with the escape of holes to native defects in GaAsSb. The nonradiative process involving such carrier traps 
has a secondary impact due to a low density of states and the overall lower probability of this process.

Acknowledgments We would like to acknowledge the National Science Centre of Poland for support within Grant No. 2014/15/B/ST7/04663.

Open Access This article is distributed under the terms of the Creative Commons Attribution 4.0 International License (http://creativecommons.org/licenses/by/4.0/), which permits unrestricted use, distribution, and reproduction in any medium, provided you give appropriate credit to the original author(s) and the source, provide a link to the Creative Commons license, and indicate if changes were made.

\section{References}

Bauer, A., Rößner, K., Lehnhardt, T., Kamp, M., Höfling, S., Worschech, L., Forchel, A.: Mid-infrared semiconductor heterostructure lasers for gas sensing applications. Semicond. Sci. Technol. 26, 014032 (2010)

Bir, G.L., Pikus, G.E., Shelnitz, P., Louvish, D. (1976) Symmetry and strain-induced effects in semiconductors. Wiley, Israel Program for Scientific Translations; Distributed by Wiley, New York; Jerusalem; London; Chichester

Dallner, M., Hau, F., Höfling, S., Kamp, M.: InAs-based interband-cascade-lasers emitting around $7 \mu \mathrm{m}$ with threshold current densities below $1 \mathrm{kA} / \mathrm{cm} 2$ at room temperature. Appl. Phys. Lett. 106, 041108 (2015)

Dong, L., Li, C., Sanchez, N.P., Gluszek, A.K., Griffin, R.J., Tittel, F.K.: Compact CH4 sensor system based on a continuous-wave, low power consumption, room temperature interband cascade laser. Appl. Phys. Lett. 108, 011106 (2016)

Dyksik, M., Motyka, M., Sęk, G., Misiewicz, J., Dallner, M., Weih, R., Kamp, M., Höfling, S.: Submonolayer uniformity of type II InAs/GaInSb W-shaped quantum wells probed by full-wafer photoluminescence mapping in the mid-infrared spectral range. Nanoscale Res. Lett. 10, 402 (2015)

Dyksik, M., Motyka, M., Rudno-Rudziński, W., Sęk, G., Misiewicz, J., Pucicki, D., Kosiel, K., Sankowska, I., Kubacka-Traczyk, J., Bugajski, M.: Optical properties of active regions in terahertz quantum cascade lasers. J. Infrared Millim. Terahertz Waves 37, 710-719 (2016)

Fang, Y., Wang, L., Sun, Q., Lu, T., Deng, Z., Ma, Z., Jiang, Y., Jia, H., Wang, W., Zhou, J., Chen, H.: Investigation of temperature-dependent photoluminescence in multi-quantum wells. Sci. Rep. 5, 12718 (2015)

Firsov, D.D., Komkov, O.S.: Photomodulation Fourier transform infrared spectroscopy of semiconductor structures: features of phase correction and application of method. Tech. Phys. Lett. 39, 1071-1073 (2013)

Hosea, T.J.C., Merrick, M., Murdin, B.N.: A new Fourier transform photo-modulation spectroscopic technique for narrow band-gap materials in the mid- to far-infrared. Phys. Status Solidi Appl. Mater. Sci. 202, 1233-1243 (2005)

Jiang, Y., Li, L., Tian, Z., Ye, H., Zhao, L., Yang, R.Q., Mishima, T.D., Santos, M.B., Johnson, M.B., Mansour, K.: Electrically widely tunable interband cascade lasers. J. Appl. Phys. 115, 113101 (2014)

Kim, C.S., Kim, M., Abell, J., Bewley, W.W., Merritt, C.D., Canedy, C.L., Vurgaftman, I., Meyer, J.R.: Mid-infrared distributed-feedback interband cascade lasers with continuous-wave single-mode emission to $80^{\circ} \mathrm{C}$. Appl. Phys. Lett. 101, 10-13 (2012)

Kujala, J., Segercrantz, N., Tuomisto, F., Slotte, J.: Native point defects in GaSb. J. Appl. Phys. 116, 143508 (2014)

Li, L., Ye, H., Jiang, Y., Yang, R.Q., Keay, J.C., Mishima, T.D., Santos, M.B., Johnson, M.B.: MBE-grown long-wavelength interband cascade lasers on InAs substrates. J. Cryst. Growth 425, 369-372 (2015)

Liu, M.H., Wang, Y.H., Houng, M.P.: Carrier transport in InAs/AlSb/GaSb interband tunneling structures. J. Appl. Phys. 74, 6222-6226 (1993)

Longenbach, K.F., Luo, L.F., Wang, W.I.: Resonant interband tunneling in InAs/GaSb/AlSb/InAs and GaSb/InAs/AlSb/GaSb heterostructures. Appl. Phys. Lett. 57, 1554 (1990)

Lundqvist, S., Kluczynski, P., Weih, R., von Edlinger, M., Nähle, L., Fischer, M., Bauer, A., Höfling, S., Koeth, J.: Sensing of formaldehyde using a distributed feedback interband cascade laser emitting around 3493 nm. Appl. Opt. 51, 6009-6013 (2012) 
Meyer, J.R., Hoffman, C.A., Bartoli, F.J., Ram-Mohan, L.R.: Type-II quantum-well lasers for the midwavelength infrared. Appl. Phys. Lett. 67, 757 (1995)

Motyka, M., Misiewicz, J.: Fast differential reflectance spectroscopy of semiconductor structures for infrared applications by using Fourier transform spectrometer. Appl. Phys. Express. 3, 112401 (2010)

Motyka, M., Sęk, G., Ryczko, K., Misiewicz, J., Lehnhardt, T., Höfling, S., Forchel, A.: Optical properties of GaSb-based type II quantum wells as the active region of midinfrared interband cascade lasers for gas sensing applications. Appl. Phys. Lett. 94, 15-18 (2009a)

Motyka, M., Sęk, G., Misiewicz, J., Bauer, A., Dallner, M., Höfling, S., Forchel, A.: Fourier transformed photoreflectance and photoluminescence of mid infrared GaSb-based type II quantum wells. Appl. Phys. Express. 2, 126505 (2009b)

Motyka, M., Ryczko, K., Sęk, G., Janiak, F., Misiewicz, J., Bauer, A., Höfling, S., Forchel, A.: Type II quantum wells on GaSb substrate designed for laser-based gas sensing applications in a broad range of mid infrared. Opt. Mater. 34, 1107-1111 (2012)

Ohtani, K., Ohno, H.: An InAs-based intersubband quantum cascade laser. Japanese J. Appl. Physics, Part 2 Lett. 41, 1279-1281 (2002)

Pierscinski, K., Pierscinska, D., Szabra, D., Nowakowski, M., Wojtas, J., Mikolajczyk, J., Bielecki, Z., Bugajski, M. (2014) Time resolved FTIR study of spectral tuning and thermal dynamics of mid-IR QCLs. In: Panajotov, K., Sciamanna, M., Valle, A., and Michalzik, R. (eds.) Semiconductor Lasers and Laser Dynamics VI. p. 91341L. SPIE-INT Soc Optical Engineering, 1000 20th St, PO Box 10, Bellingham, WA 98227-0010 USA

Ryczko, K., Sęk, G., Misiewicz, J.: Eight-band k·p modeling of InAs/InGaAsSb Type-II W-design quantum well structures for interband cascade lasers emitting in a broad range of mid infrared. J. Appl. Phys. 114, 223519 (2013)

Sęk, G., Janiak, F., Motyka, M., Ryczko, K., Misiewicz, J., Bauer, A., Höfling, S., Forchel, A.: Carrier loss mechanisms in type II quantum wells for the active region of GaSb-based mid-infrared interband cascade lasers. Opt. Mater. 33, 1817-1819 (2011)

Shao, J., Lu, W., Yue, F., Lü, X., Huang, W., Li, Z., Guo, S., Chu, J.: Photoreflectance spectroscopy with a step-scan Fourier-transform infrared spectrometer: technique and applications. Rev. Sci. Instrum. 78, 013111 (2007)

Suhandi, A., Feranie, S., Arifin, P.: Study of electrical properties of GaAs 1-x Sb x thin film grown by vertical-MOCVD using TMGa, TDMAAs, and TDMASb. J. Mater. Sci. Eng. A. 1, 199-203 (2011)

Thomas, J.W.: Partial Differential Equations, Finite Difference Methods. Springer, New York (1995)

Tian, Z., Li, L., Ye, H., Yang, R.Q., Mishima, T.D., Santos, M.B., Johnson, M.B.: InAs-based interband cascade lasers with emission wavelength at $10.4 \mu \mathrm{m}$. Electron. Lett. 48, 113-114 (2012)

Tittel, F.K.: Current status of midinfrared quantum and interband cascade lasers for clinical breath analysis. Opt. Eng. 49, 111123 (2010)

Von Edlinger, M., Scheuermann, J., Weih, R., Zimmermann, C., Nähle, L., Fischer, M., Koeth, J., Höfling, S., Kamp, M.: Monomode interband cascade lasers at $5.2 \mu \mathrm{m}$ for nitric oxide sensing. IEEE Photonics Technol. Lett. 26, 480-482 (2014)

Vurgaftman, I., Meyer, J.R., Ram-Mohan, L.R.: Band parameters for III-V compound semiconductors and their alloys. J. Appl. Phys. 89, 5815 (2001)

Vurgaftman, I., Bewley, W.W., Canedy, C.L., Kim, C.S., Kim, M., Merritt, C.D., Abell, J., Lindle, J.R., Meyer, J.R.: Rebalancing of internally generated carriers for mid-infrared interband cascade lasers with very low power consumption. Nat. Commun. 2, 585 (2011)

Yang, R.Q.: Infrared laser based on intersubband transitions in quantum wells. Superlattices Microstruct. 17, 77-83 (1995) 